\title{
INDEX THEORY FOR SINGULAR QUADRATIC FUNCTIONALS IN THE CALCULUS OF VARIATIONS ${ }^{1}$
}

\author{
BY JUNIOR STEIN ${ }^{2}$
}

Communicated by Everett Pitcher, April 13, 1973

1. Introduction. Let $P, Q$, and $R$ be real-valued $n \times n$ matrix functions defined on the interval $[a, b)$. Assume that $P, Q$, and $R$ are continuous on $[a, b)$ and that $P(t)$ and $R(t)$ are symmetric matrices for each $t$ in $[a, b)$. We do not assume that $Q$ is symmetric. Also assume that $R$ has the property that its value for any $t$ in $[a, b)$ is positive definite, that is, $v^{*} R(t) v>0$ for all $n$-vectors $v \neq 0$ and for each $t$ in $[a, b)$. Let

$$
\begin{aligned}
\left.J(x, y)\right|_{e_{1}} ^{e_{2}}= & \int_{e_{1}}^{e_{2}}\left[\dot{x}^{*}(t) R(t) \dot{y}(t)+x^{*}(t) Q(t) \dot{y}(t)+\dot{x}^{*}(t) Q^{*}(t) y(t)\right. \\
& \left.+x^{*}(t) R(t) y(t)\right] d t \quad\left(a \leqq e_{1} \leqq e_{2}<b\right),
\end{aligned}
$$

for $x$ and $y$ in the class $A$ of vector-valued functions described below. Also let

$$
\begin{aligned}
J_{e}(x, y)=\left.J(x, y)\right|_{a} ^{e}, & J_{e}(x)=J_{e}(x, x), \\
J(x, y)=\liminf _{e \rightarrow b-} J_{e}(x, y), & J(x)=\liminf _{e \rightarrow b-} J_{e}(x)
\end{aligned}
$$

for $x$ and $y$ in $A$. The class $A$ is the set of vector-valued functions $x^{*}(t)=$ $\left(x_{1}(t), \ldots, x_{n}(t)\right), a \leqq t \leqq b$, satisfying

(i) $x(t)$ is continuous on the interval $[a, b]$ and $x(a)=x(b)=0$,

(ii) $x(t)$ is absolutely continuous and $\dot{x}^{*}(t) \dot{x}(t)$ is Lebesgue integrable on each closed subinterval of $[a, b)$. $A$ is a vector space of functions.

$J$ is said to be singular at a point $t$ in $[a, b]$ if the determinant of $R(t)$ is zero or not defined. The point $t=b$ is a singular point in this paper.

2. Preliminaries. What is presented here is part of a quadratic form theory developed and used extensively by Hestenes [3], [4]. Let $Q(x)$

AMS(MOS) subject classifications (1970). Primary 49B10, 34C10; Secondary 58E99.

Key words and phrases. Singular quadratic functionals, singular differential equations, index theory of quadratic forms.

${ }^{1}$ The author is indebted to Professor Magnus R. Hestenes for suggesting this problem and for his suggestions in its preparation.

${ }^{2}$ This is to acknowledge the partial support of the author by the U.S. Army Research Office at Durham under Grant DA-31-124-ARO(D)-355 and under Grant DA-ARO-D-31124-71-G18. Reproduction in whole or in part is permitted for any purpose of the United States Government. 
be a quadratic functional defined on a vector space $V$ and let $Q(x, y)$ be its associated symmetric bilinear functional. Two vectors $x$ and $y$ in $V$ are said to be $Q$-orthogonal whenever $Q(x, y)=0$. A vector $x$ is said to be $Q$-orthogonal to a subset $S$ of $V$ whenever $Q(x, y)=0$ for every $y$ in $S$. By the $Q$-orthogonal complement $S^{Q}$ of the set $S$ in $V$ is meant the set of all vectors $x$ in $V$ that are $Q$-orthogonal to $S$. $S^{Q}$ is a subspace of $V$. A vector in $S$ that is $Q$-orthogonal to $S$ is called a $Q$-null vector of $S$. The intersection $S \cap S^{Q}$ is the set of $Q$-null vectors of $S$ and is usually denoted by $S_{0}$. If $S$ is a subspace of $V$, then so is $S_{0}$.

Let $S$ be any subspace in $V$. We define the nullity $n(S)$ of $Q$ on $S$ or more simply the $Q$-nullity of $S$ to be the dimension of the subspace $S_{0}=S \cap S^{Q}$ of $Q$-null vectors in $S$. We define the signature $s(S)$ of $Q$ on $S$, the index of $Q$ on $S$, or the $Q$-signature of $S$ to be the dimension of a maximal subspace $M$ of $S$ on which $Q<0$ if this dimension is finite. If no such finite dimensional space exists, we set $s(S)=\infty$. By $Q<0$ on $M$ we mean that $Q(x)<0$ for each nonzero $x$ in $M$. It turns out that the dimension $s(S)$ of $M$ is independent of the choice of $M$ so that the notion of signature is well defined.

THEOREM 2.1. If the $Q$-signature of $S$ is finite where $S$ is a subspace of $V$, then it is given by one of the following quantities:

(i) the dimension of a maximal subspace $M$ in $S$ on which $Q<0$;

(ii) the dimension of a maximal subspace $M$ of $S$ on which $Q \leqq 0$ and having $M \cap S_{0}=0$;

(iii) the dimension of a minimal subspace $M$ of $S$ such that $Q \geqq 0$ on $S \cap M^{Q}$;

(iv) the least integer $k$ such that there exist $k$ linear functionals $L_{1}, \ldots, L_{k}$ on $S$ with the property that $Q(x) \geqq 0$ for all $x$ in $S$ satisfying the conditions $L_{\alpha}(x)=0(\alpha=1,2, \ldots, k)$.

3. Results. The main purpose of this paper is to announce the results presented in this section. The details and more results are to appear elsewhere.

The definition of a singular conjugate point is found in Tomastik $[7$, p. 61] and Chellevold [1, p. 333]. It extends the definition of Morse and Leighton $[5$, p. 253], who treated the case $n=1$. For $a \leqq e \leqq b$ let $A(e)=\{x \in A: x(t)=0$ for $e \leqq t \leqq b\}$, where $A$ is defined in $\S 1$ of this paper. Define the set $B$ in $A$ to be the union of the sets $A(e)$ for $a<e<b$. Observe that $B$ is actually a subspace of $A$.

THEOREM 3.1. The following conditions are equivalent for some nonnegative integer $k$ :

(i) The signature of $J$ given by (1.3) on $B$ is $k$. 
(ii) There is an $\varepsilon_{0}$ in $(a, b)$ such that $\varepsilon_{0} \leqq \varepsilon<b$ implies that the signature of $J$ given by (1.3) on $A(\varepsilon)$ is $k$.

(iii) The point a has exactly a finite number $k$ of nonsingular conjugate points on $a<t<b$.

(iv) The point $b$ has exactly a finite number $k$ of singular conjugate points on $a<t<b$.

(v) $b$ is not conjugate to $b$.

Theorem 3.1 above contains Theorem 4.4, p. 337, of Chellevold [1]. Let $U(t)$ be a conjugate system satisfying Euler's equation

$$
\left[R(t) \dot{U}(t)+Q^{*}(t) U(t)\right]^{\prime}=[Q(t) \dot{U}(t)+P(t) U(t)]
$$

and the conditions $U(a)=0, \dot{U}(a)=I$, det $U(t) \neq 0$ for $t$ near $b$. Let us remark that there are $J$ 's which do not possess such conjugate systems. For $y$ in $A$ and for $t$ near $b$ set

$$
S[y(t), a]=y^{*}(t)\left\{\left[R(t) \dot{U}(t)+Q^{*}(t) U(t)\right] U^{-1}(t)\right\} y(t) .
$$

Let $D$ be a subspace in $A$ satisfying $B \subseteq D \subseteq A$. The condition that $\lim \inf _{t \rightarrow b-} S[y(t), a] \geqq 0$ for each $y$ in $D$ satisfying $\left.\lim \inf _{t \rightarrow b-} J(y)\right|_{a} ^{t}<\infty$ is called the singularity condition relative to $D$ and belonging to $[a, b]$.

THeOREM 3.2. Assume that $s(B)$ is finite. Let $D$ be any subspace with $B \subseteq D \subseteq A$. Let $C$ be a subspace in $B$ maximal relative to having $J<0$. Let $C^{J}=\{x \in A: J(x, y)=0$ for all $y$ in $C\}$. The following conditions are equivalent:

(i) If $x$ is in $D \cap C^{J}$, then $J(x)<\infty$ implies $\lim _{\inf _{e \rightarrow b-}} S[x(e), a] \geqq 0$.

(ii) If $x$ is in $D \cap C^{J}$, then $J(x) \geqq 0$.

(iii) The singularity condition relative to $D$ holds; that is, if $x$ is in $D$, then $J(x)<\infty$ implies $\lim \inf _{e \rightarrow b-} S[x(e), a] \geqq 0$.

THEOREM 3.3. Suppose that $J(x, y)=\lim _{\inf _{e \rightarrow b-}} J_{e}(x, y)$ is bilinear on the subspace $D$ where $B \subseteq D \subseteq A$. Assume that $s(B)$ is finite. Let $C$ be a subspace in B maximal relative to having $J<0$. Let $C^{J}=\{x \in A: J(x, y)=$ 0 for all $y$ in $C\}$. Then $s(D)=s(B)$ if and only if $x$ in $C^{J} \cap D$ implies $J(x) \geqq 0$.

Corollary. If $J$ is bilinear on the subspace $D$ with $B \subseteq D \subseteq A$ and $s(B)$ is finite, then $s(D)=s(B)$ if and only if the singularity condition relative to $D$ and belonging to $[a, b]$ holds.

The next theorem generalizes Theorems 2.3, 4.1, and 5.1 of Tomastik [7].

THEOREM 3.4. There is a subspace $C$ of finite dimension $k$ in $B$ with $C$ maximal relative to having $J<0$ and $J \geqq 0$ on $C^{J} \cap D$ holds for a subspace 
$D$ with $B \subseteq D \subseteq A$ if and only if there are $k$ conjugate points to $b$ in $(a, b]$ and the singularity condition relative to $D$ and belonging to $[a, b]$ is satisfied.

COROLlary. There is a subspace $C$ of finite dimension $k$ in $B$ with $C$ maximal relative to having $J<0$ and $J \geqq 0$ on $C^{J}$ holds if and only if there are $k$ conjugate points to $b$ in $(a, b]$ and the singularity condition relative to $A$ and belonging to $[a, b]$ is satisfied.

Corollary. For any subspace $D$ with $B \subseteq D \subseteq A, J \geqq 0$ on $D$ holds if and only if there are no conjugate points to $b$ in $(a, b]$ and the singularity condition relative to $D$ and belonging to $[a, b]$ is satisfied.

\section{BIBLIOGRAPHY}

1. J. O. Chellevold, Conjugate points of singular quadratic functionals for $n$ dependent variables, Proc. Iowa Acad. Sci. 59 (1952), 331-337. MR 14, 769.

2. G. H. Hardy, J. E. Littlewood and G. Pólya, Inequalities, Cambridge Univ. Press, New York, 1934.

3. M. R. Hestenes, Applications of the theory of quadratic forms in Hilbert space to the calculus of variations, Pacific J. Math. 1 (1951), 525-581. MR 13, 759.

4. - Quadratic form theory and analysis, Lecture Notes, Math. Dept. Reading Room, University of California, Los Angeles, Calif., 1967.

5. M. Morse and W. Leighton, Singular quadratic functionals, Trans. Amer. Math. Soc. 40 (1936), 252-286.

6. J. Stein, Singular quadratic functionals, Dissertation, University of California, Los Angeles, Calif., 1971.

7. E. C. Tomastik, Singular quadratic functionals of $n$ dependent variables, Trans. Amer. Math. Soc. 124 (1966), 60-76. MR 33 \#4743.

Department of Mathematics, The University of Toledo, Toledo, Ohio 43606 